\title{
Group A streptococcal toxic shock syndrome associated with pelvic peritonitis following diagnostic hysteroscopy in a healthy woman: a case report and review
}

\author{
Kalsang Bhatia • Simon Hill
}

Received: 9 March 2008 / Accepted: 20 April 2008/ Published online: 20 May 2008

(C) Springer-Verlag 2008

\begin{abstract}
We describe a case of group A streptococcal toxic shock syndrome (TSS) associated with pelvic peritonitis, occurring after a diagnostic hysteroscopy and curettage in a healthy woman. At laparotomy, performed to rule out bowel perforation, the diagnosis of pelvic inflammatory disease and pelvic peritonitis was confirmed. Her general condition deteriorated postoperatively needing supportive care in the intensive care unit, followed by gradual recovery. Although it is difficult to avoid a laparotomy in most cases following a surgical procedure, it is important to consider the diagnosis of streptococcal TSS associated with pelvic peritonitis from ascending sepsis in rapid onset, severely shocked cases.
\end{abstract}

Keywords Group A streptococcal · Toxic shock syndrome · Pelvic peritonitis $\cdot$ Hysteroscopy $\cdot$ Curettage

\section{Introduction}

Hysteroscopy with curettage to obtain endometrial biopsy is a common gynaecological procedure and is relatively free of any serious complications. Ascending infection with pelvic inflammatory disease (PID) after any intrauterine procedure is a recognised risk, and hence, it is a good practice to screen and treat any lower genital tract infections in high risk and symptomatic women prior to the procedure.

\footnotetext{
K. Bhatia $\cdot$ S. Hill

Royal Blackburn Hospital,

Haslingden Road,

Blackburn, Lancashire, UK

K. Bhatia $(\bowtie)$

7 Whinney Hey's Road,

Blackpool FY3 8NP Lancashire, UK

e-mail: Naviapo@aol.com
}

Group A streptococcal (GAS) toxic shock syndrome (TSS) associated with pelvic peritonitis following minor uterine manipulative procedures is however very rare and most reported cases have been in relation to pregnancy and puerperium [1-4]. Nyirjesy et al. reported a case with streptococcal TSS following laparoscopic tubal ligation 5 weeks postpartum [5]. Mourton and Rich published the first report of a similar case occurring after an office endometrial biopsy [6].

Group A streptococcus or Streptococcus pyogenes may cause infections ranging from mild pharyngitis or impetigo to severe life-threatening conditions such as necrotising fascitis and toxic shock syndrome. Invasive GAS infection is associated with toxic shock syndrome in $8-14 \%$ cases, resulting in shock and multi-organ failure soon after onset of symptoms, and is associated with a mortality of $33 \%$ despite antibiotics and supportive therapy $[1,7]$. It has been reported that over the last two decades there has been an increase in the incidence of invasive GAS disease. This trend may be related to the increasing prevalence of strains with specific M-proteins (M-1 and M-3) [1].

We report a case of GAS toxic shock-like syndrome associated with pelvic peritonitis occurring after a diagnostic hysteroscopy with uterine curettage in a healthy woman, emphasising on early recognition of this life-threatening infection, and review on the current concept of pathogenesis and management of streptococcal TSS.

\section{The case}

A 51-year-old, fit and healthy lady was referred to our gynaecological clinic with irregular and heavy periods. There was no history of abnormal vaginal discharge or postcoital bleeding. Clinical findings were unremarkable 
except for a cervical ectropion; high vaginal and endocervical swabs taken at that time were reported as negative. A recent cervical smear was also negative. Three weeks later she underwent a diagnostic hysteroscopy and endometrial biopsy as day case. A red cervix reported as an ectropion was noted; uterine cavity was normal with secretory endometrium and normal cervical canal. The procedure was uneventful and she was discharged soon after.

Approximately $3.5 \mathrm{~h}$ later, she developed acute upper abdominal pain and bilious vomiting. The abdominal pain soon became generalised and she developed watery stools as well. On admission, she was shocked with a blood pressure of $70 / 40 \mathrm{mmHg}$, a pulse rate of $130 \mathrm{bpm}$ and was severely dehydrated with a body temperature of $38.5^{\circ} \mathrm{C}$. She was also hypoxic (oxygen saturation on air of 91\%) and tachypnoeic although she was alert and responded to verbal commands. The abdomen was not unduly distended but there was generalised tenderness, guarding and rigidity. Bowel sounds were normal. There was generalised tenderness in the pelvis on bimanual examination with some vaginal discharge and vaginal swabs were repeated. Radiological examination of the chest and abdomen was unremarkable with no evidence of gas or fluid levels under the diaphragm. Blood tests on admission confirmed sepsis with raised white cell count and C-reactive protein; there was also evidence of disseminated intravascular coagulation. Arterial blood gases showed that she was acidotic.

She was resuscitated with oxygen, intravenous fluids and inotropes and commenced on intravenous antibiotics in the form of ceftriaxone and metronidazole. It then came to light that a high vaginal swab repeated by her general practitioner 3 days before her operation showed $S$. pyogenes sensitive to penicillin. After consulting a consultant microbiologist, the antibiotic regime was changed to benzyl penicillin $2.4 \mathrm{mg} / 6 \mathrm{~h}$, clindamycin $600 \mathrm{mg} / 6 \mathrm{~h}$ and gentamicin $320 \mathrm{mg}$ as single daily dose.

Her general condition remained the same despite 5-6 h of continued resuscitation, and it was then decided to do a laparotomy to rule out peritonitis secondary to bowel perforation. At laparotomy, the peritoneal cavity was filled with turbid fluid containing fibrinous exudates. The serosal surface of about $100 \mathrm{~cm}$ of small bowel lying in the pelvis appeared inflamed. The fimbrial ends of both the fallopian tubes were inflamed and both ovaries were covered with exudates. However, the uterus was intact with no perforation. Other abdominal organs were also normal. A diagnosis of acute pelvic peritonitis was made and after obtaining some peritoneal fluid for microbiology, peritoneal lavage was performed and the abdomen closed.

The patient's general condition deteriorated soon after laparotomy with worsening metabolic acidosis, disseminated intravascular coagulation and sepsis, requiring transfer to the intensive care unit. She needed ventilation, inotropic support and transfusion with blood and fresh frozen plasma. Ventilation and inotropic support was weaned after $48 \mathrm{~h}$ and she was maintained on the same antibiotics although she remained pyrexial till the fifth postoperative day. Blood cultures grew group A streptococci although the peritoneal fluid did not reveal any organism. Thromboprophylaxis was given until she was discharged home on the seventh postoperative day.

\section{Discussion}

Streptococcal TSS is an uncommon but life-threatening infection, which may be encountered by any gynaecologist. The diagnosis should be considered in any case with rapid onset of shock associated with multiple organ failure; GAS should be isolated from normally sterile/non-sterile body sites $[2,8]$. The criteria for the diagnosis of streptococcal TSS have been previously published and these are shown in Table $1[2,9]$. Our patient fulfilled the criteria for diagnosis.

The knowledge of pathogenesis of invasive streptococcal infection is essential to help understand the course of this disease and its management. GAS produces virulence factors such as streptolysin $\mathrm{O}$, superantigens and invasins. The streptococcal pyrogenic exotoxins (a type of superantigens) are polypeptides produced by certain group A streptococci which have the capacity to stimulate the immune system and thereby induce a cascade of cytokines. The action of cytokines is assumed to be responsible for the induction of TSS. Superantigens such as streptococcal pyrogenic exotoxins (similar to Staphylococcus endotoxin responsible for staphylococcal TSS) do not require processing by antigen-presenting cells before they can stimulate $T$ cells. These superantigens cause an aberrant proliferation of specific $\mathrm{T}$ cell subsets and directly activate thousands of

Table 1 Diagnostic criteria for group A streptococcal TSS [2, 9]

Criteria

A. Isolation of group A streptococcus:

(1) From a sterile site

(2) From a non-sterile body site

B. Clinical signs of severity

(1) Hypotension

(2) Clinical and laboratory abnormalities (requires two or more of the following):

(a) Renal impairment

(b) Coagulopathy

(c) Liver abnormalities

(d) Acute respiratory distress syndrome

(e) Extensive tissue necrosis, i.e. necrotising fasciitis

(f) Erythematous rash

Definite case: $\mathrm{A} 1+\mathrm{B}(1+2)$

Probable case: $\mathrm{A} 2+\mathrm{B}(1+2)$ 
times more $\mathrm{T}$ cells (up to $25 \%$ of total $\mathrm{T}$ cell population) than conventional antigens, to produce an accelerated cascade of cytokines such as interleukins and tumour necrosis factor with rapid clinical sequelae. It is at this stage that antibiotics alone may not be able to control the GAS infection $[1,2,8]$.

The therapeutic management of severe GAS infections requires high doses of intravenous antibiotics, including a beta-lactam agent (penicillin) and clindamycin. Group A streptococcal infections remain sensitive to penicillin, and patients allergic to penicillin may be treated by firstgeneration cephalosporin or vancomycin. Clindamycin has been recommended in addition to penicillin for all patients. Evidences from in vitro observations and animal studies indicate that clindamycin may be more effective than penicillin at inhibiting these organisms when they are present in high inocula and not dividing rapidly $[1,2]$. Harry Eagle first showed that streptococci present at high inoculum $(>107 \mathrm{cfu} / \mathrm{ml})$ are very slowly killed by penicillin. Animal models suggest that clindamycin, with its inhibition of protein synthesis, has greater activity than penicillin against organisms in a stationary phase of growth and may reduce production of toxins and TNF. This effect of clindamycin is called the 'Eagle effect' [2, 10]. Clindamycin alone is not recommended because some strains are resistant, especially in regions where macrolides are used extensively in the community. Invasive streptococcal TSS is fulminating and may not respond to delayed antibiotic therapy; therefore, it is obvious that antibiotics and aggressive intensive care treatment should be started as early as possible. Case-control studies on the use of immunoglobulin preparations in streptococcal TSS show a reduction in mortality from $67 \%$ to $34 \%$ [1, 2, 11]. Despite the absence of randomised controlled trials, most experts advocate the use of immunoglobulins in severely shocked patients - preferably early in the disease course $[1,2,12]$.

When a patient presents soon after a surgical procedure, which has a potential for bowel injury, the diagnosis may be delayed until laparotomy as seen in this case. The index of suspicion for secondary peritonitis following unrecognised bowel trauma was high and therefore, it is often difficult to avoid a laparotomy. A diagnostic laparoscopy however could have been more useful in ruling out uterine perforation and perhaps shortened the duration of her illness.

Unfortunately in this case, what is clear is that a proper preoperative assessment of the patient's new symptoms/ episodes on the day of procedure and indeed proper communication between primary and secondary caregivers with appropriate action may have prevented this lifethreatening complication. It was not known to us that her lower genital tract was colonised with GAS when the procedure was performed. If this were known, the operation would have been deferred until her symptoms have been reassessed after antibiotics or the patient would have been given intraoperative and postoperative antibiotics. When seen in the outpatient clinic ( 3 weeks prior), she did not complain of any vaginal discharge and the preoperative vaginal swabs were taken opportunistically in view of the appearance of her cervix, albeit her age. Cleansing of the vagina with betadine during the procedure made it difficult to assess the presence of any vaginal discharge and the inflamed cervix was thought to be an ectropion. If there was any doubt at that point, a prophylactic dose of intraoperative antibiotics may have prevented this complication.

GAS is normally easy to grow requiring no special culture media or care and can be easily recognised on microscopy appearing as gram-positive chains. There was no undue delay in transporting the specimens from the clinic and hence difficult to explain why the swabs were negative. It is therefore very likely that colonisation with GAS in this case may have occurred in the weeks following her outpatient visit, when the patient consulted her general practitioner for her new symptoms of vaginal discharge.

It is not uncommon to have negative culture of peritoneal fluid in cases of peritonitis and this is often due to insensitive culture methods [13]. When there is associated PID, the culture is more likely to yield positive results if the specimen is taken from the pouch of Douglas in close proximity with the fimbrial ends of fallopian tubes rather than from the general peritoneal cavity. The preferred technique for culturing peritoneal fluid is by inoculating $5 \mathrm{ml}$ into blood culture bottles; this has been shown to increase sensitivity from $42 \%$ to $91 \%$ [13]. These methods may not have been adhered to in this case.

In summary, although rare, gynaecologists need to be aware of the diagnosis of streptococcal TSS associated with pelvic peritonitis in patients presenting with rapid onset shock following any intrauterine procedures. A proper preoperative assessment of new symptoms and administration of intraoperative antibiotics when in doubt will help prevent such life-threatening complications.

\section{References}

1. Crum NF, Chun HM, Gaylord TG et al (2002) Group A streptococcal toxic shock syndrome developing in the third trimester of pregnancy. Infect Dis Obstet Gynecol 10:209-216

2. Schummer W, Schummer C (2002) Two cases of delayed diagnosis of postpartal streptococcal toxic shock syndrome. Infect Dis Obstet Gynecol 10:217-222

3. Palep-Singh M, Jayaprakasan J, Hopkisson JF (2007) Peripartum group A streptococcal sepsis: a case report. J Reprod Med 52 (10):977-978 Oct

4. Okumura K, Schroff R, Campbell R, Nishioka L, Elster E (2004) Group A streptococcal puerperal sepsis with retroperitoneal 
involvement developing in a late postpartum woman: case report. Am Surg 70(8):730-732 Aug

5. Nyirjesy P, Jones RS, Chatwani A, Zinner ES, Axelrod P (1994) Streptococcal toxic shock-like syndrome as an unusual complication of laparoscopic tubal ligation. A case report. J Reprod Med 39(8):649-651 Aug

6. Mourton S, Rich W (2006) Group A streptococcal toxic shock syndrome after an office endometrial biopsy: a case report. J Reprod Med 51(8):665-668 Aug

7. Baxter F, Mc Chesney J (2000) Severe group A streptococcal infection and streptococcal toxic shock syndrome. Can J Anaesth 47(11):1129-1140 Nov, Review

8. Stegmayr B, Bjorck S, Holm S et al (1992) Septic shock induced by group A streptococcal infection: clinical and therapeutic aspects. Scand J Infect Dis 24:589-597

9. The Working Group on Severe Streptococcal Infections (1993) Defining the group A streptococcal toxic shock syndrome: rationale and consensus definition. J Am Med Assoc 269: 390-391

10. Stevens DL, Gibbons AE, Bergstrom R et al (1988) The Eagle effect revisited: efficacy of clindamycin, erythromycin, and penicillin in the treatment of streptococcal myositis. J Infect Dis 158:23-28

11. Perez CM, Kubak BM, Cryer HG et al (1997) Adjunctive treatment of streptococcal toxic shock syndrome using intravenous immunoglobulin: case report and review. Am J Med 102:111-113

12. Darenberg J, Ihendyane N, Sjölin J, Aufwerber E, Haidl S, Follin P, Andersson J, Norrby-Teglund A, StreptIg Study Group (2003) Intravenous immunoglobulin $G$ therapy in streptococcal toxic shock syndrome: a European randomized, double-blind, placebocontrolled trial. Clin Infect Dis 37(3):341-343 Aug 1, Comment

13. Runyon BA, Umland ET, Merlin T (1987) Inoculation of blood culture bottles with ascitic fluid. Arch Intern Med 147:73-75 